\title{
Diagnosis of giant cell arteritis in a large urban district hospital
}

\author{
Authors: Maria Jasim ${ }^{A}$ and Tazeen J Ahmed ${ }^{B}$
}

\section{Background}

Giant cell arteritis (GCA) is a rare disease and the commonest of the vasculitides. Given the risk of sight loss, it is also a rheumatological emergency. The 1990 American College of Rheumatology (ACR) classification guidelines ${ }^{1}$ are often used as a guide to diagnosis, with an emphasis on age, erythrocyte sedimentation rate (ESR) and new headache. With new diagnostic modalities such as ultrasound and computed tomography-positron emission tomography (CT-PET) scanning and the introduction of tocilizumab as a new therapeutic agent, we felt it important to look at current clinical diagnosis of this condition.

\section{Methods}

Patient records coded with a diagnosis of giant cell arteritis, temporal arteritis or a temporal artery biopsy procedure over the period of June 2017 - June 2018 were identified from the electronic patient record. Forty-four patients were identified; of these, 24 were historical diagnoses. The data were collected retrospectively and analysed using MS Excel 2010. The patients were classified into high- and low-risk categories using the local pathway criteria and a recently published gradation system.

\section{Results and discussion}

Twenty patients were identified in this 1-year period. The low number may be a reflection of local clinical coding. The average age was 72.6 years, $90 \%$ were female and the commonest ethnicity was white British (30\%). Twenty-five per cent were seen by both neurology and rheumatology. Forty-five per cent were only seen by a neurologist. Fifteen per cent saw neither rheumatology nor neurology.

Only one patient had a positive temporal artery biopsy (17 out of 20 underwent biopsy), so diagnoses were made clinically. Eighty-five per cent presented with headache, $65 \%$ had scalp tenderness, $50 \%$ had an ESR over 50 and $100 \%$ were aged over 50 on presentation. Fifty-five per cent had visual disturbance on presentation. Sixty per cent had myalgic symptoms ( 14 out of

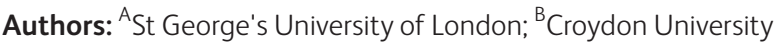
Hospital
20 documented). Thirty-five per cent had systemic symptoms (eight out of 20 documented). Fifty per cent without documentation of systemic symptoms and $83 \%$ without presence of polymyalgia-like symptoms documented had also not seen a rheumatologist.

Using the local guidelines, seven were graded as high risk (of these, two were given a GCA diagnosis), one as low risk (not diagnosed with GCA) and 12 as 'clinically possible' (eight diagnosed with GCA). Using the literary guidelines, 14 were graded as high risk (seven diagnosed with GCA) and six as low risk. Thirteen would have been classified with GCA according to the 1990 ACR guidelines, ${ }^{1}$ of whom seven were diagnosed with GCA. Further analysis is limited by the small sample size.

\section{Conclusion}

The number of patients assessed for GCA in this time period is lower than would be expected for the catchment population. Patients were seen by a variety of specialties, with not all following the local GCA pathway. The grading systems gave varying outcomes, showing the need for clinical acumen and specialist rheumatological input in the diagnosis of GCA. This is shown to be especially important as the majority of biopsies did not rule out temporal arteritis.

\section{Reference}

1 Hunder GG, Bloch DA, Michel BA et al. The American College of Rheumatology 1990 Criteria for the classification of giant cell arteritis. Arthritis Rheum 1190;33:1122-8. 